\title{
Some remarks on structural matrix rings and matrices with ideal entries
}

\author{
Stephan Foldes and Gerasimos Meletiou
}




\title{
SOME REMARKS ON STRUCTURAL MATRIX RINGS AND MATRICES WITH IDEAL ENTRIES
}

\author{
STEPHAN FOLDES AND GERASIMOS MELETIOU
}

Received 16 February, 2011

\begin{abstract}
Associating to each pre-order on the indices $1, \ldots, n$ the corresponding structural matrix ring, or incidence algebra, embeds the lattice of $n$-element pre-orders into the lattice of $n \times n$ matrix rings. Rings within the order-convex hull of the embedding, i.e. matrix rings that contain the ring of diagonal matrices, can be viewed as incidence algebras of ideal-valued, generalized pre-order relations. Certain conjugates of the upper or lower triangular matrix rings correspond to the various linear orderings of the indices, and the incidence algebras of partial orderings arise as intersections of such conjugate matrix rings.
\end{abstract}

2000 Mathematics Subject Classification: 06A11; 06A75; 15A09; 15A30; 15B99; 16S50; 16 U60

Keywords: structural matrix ring, incidence algebra, pre-order, quasi-order, triangular matrix, conjugation, semiring, ideal lattice, subring lattice

\section{CONJUgate SUBRings}

Rings are understood to be possibly non-commutative, and to have a unit (multiplicatively neutral) element, which is assumed to be distinct from the zero (null) element and is denoted by 1 or $I$ or a similar symbol. Subrings are understood to contain the unit element. An $n \times n$ matrix is viewed as a map defined on the set $\mathbf{n}^{2}=\{1, \ldots, n\}^{2}$. (Here $n \geq 1$ is assumed.) The ring of $n \times n$ matrices over a ring $R$ is denoted by $M_{n}(R)$.

A pre-order, also called quasi-order, is a reflexive and transitive binary relation $\lesssim$ on a set $S$, an order (or partial order) is an anti-symmetric pre-order, and a linear (or total) order is an order in which any two elements are comparable. Instead of the generic notation $\lesssim$, specific pre-orders may be denoted by other symbols such as $\theta$. Given a ring $R$ and a pre-order $\lesssim$ on $\mathbf{n}$, the structural matrix ring $M_{n}(\lesssim, R)$ over $R$ is defined by

$$
M_{n}(\lesssim, R)=\left\{A \in M_{n}(R): \forall i, j \quad A(i, j)=0 \text { unless } i \lesssim j\right\}
$$

The full matrix ring $M_{n}(R)$, the subrings of all upper triangular (respectively lower triangular), and of all diagonal matrices, are examples of structural matrix rings. 
Structural matrix rings are essentially the same as incidence algebras of finite preordered sets, although the latter term is sometimes used under the assumption that the base ring $R$ is a field [3] or that the pre-order in question is an order, possibly on an infinite set, as in [8]. Ring-theoretical properties of incidence algebras most relevant to the present context were studied in $[2,3,7,10,11]$.

The set of all pre-orders defined on any given set constitutes a lattice whose minimum is the equality relation.

Proposition 1. For any ring $R$, the map associating to each pre-order $\lesssim$ on $\mathbf{n}$ the corresponding structural matrix ring $M_{n}(\lesssim, R)$ provides an embedding of the lattice of pre-orders on $\mathbf{n}$ into the lattice of subrings of the matrix ring $M_{n}(R)$. The embedding preserves also infinite greatest lower and least upper bounds.

Proof. Preservation of greatest lower bounds is obvious. The preservation of upper bounds is a consequence of the description of the least upper bound of a family of pre-orders as the transitive closure of the least binary relation that is implied by the pre-orders in the family.

The lattice embedding described in the proposition above is generally not surjective, and for $n \geq 2$ its range is order-convex if and only if $R$ is a division ring. Section 2 will provide a description of the order-convex hull of the embedding's range.

Subrings $S$ and $T$ of $M_{n}(R)$ are said to be permutation conjugates if there is a permutation matrix $P$ such that

$$
S=\left\{P A P^{-1}: A \in T\right\}
$$

Such subrings are obviously isomorphic under the automorphism $A \mapsto P A P^{-1}$ of $M_{n}(R)$.

All the $n$ ! linear orders on the finite set $\mathbf{n}$ are isomorphic, and the isomorphisms among them are precisely the self-bijections of the underlying set $\mathbf{n}$. Consequently we have:

Proposition 2. For any pre-order $\lesssim$ on $\mathbf{n}$ and any ring $R$, the following conditions are equivalent:

(i) $\lesssim$ is a linear order,

(ii) $M_{n}(\lesssim, R)$ is a permutation conjugate of the ring of upper triangular matrices,

(iii) $M_{n}(\lesssim, R)$ is a permutation conjugate of the ring of lower triangular matrices.

The well-known fact that every partial order is the intersection of its linear extensions yields:

Proposition 3. For any pre-order $\lesssim$ on $\mathbf{n}$ and any ring $R$, the following conditions are equivalent:

(i) $\lesssim$ is an order,

(ii) $M_{n}(\lesssim, R)$ is the intersection of some permutation conjugates of the ring of upper triangular matrices, 
(iii) $M_{n}(\lesssim R)$ is the intersection of some permutation conjugates of the ring of lower triangular matrices.

These considerations were first developed, in the context of fields, in [4]. They were related to a ring property concerning one-sided and two-sided inverses in [6], addressing questions originating in [1] and [9].

\section{MATRICES WITH IDEAL ENTRIES}

The order-convex hull of the embedding provided by Proposition 1 consists obviously of those matrix rings that contain the ring of diagonal matrices. We now show that these rings can be viewed as generalized incidence algebras, corresponding to generalized relations that are the analogues of pre-order relations in a reticulated semiring-valued framework.

By a semiring we understand a set endowed with a binary operation called sum (denoted additively) and a binary operation called product (denoted multiplicatively) such that:

(i) the sum operation defines a commutative semigroup,

(ii) the product operation defines a semigroup,

(iii) both distributivity laws $a(b+c)=a b+a c$ and $(b+c) a=b a+c a$ hold for all members $a, b, c$ of the underlying set.

The set $d(R)$ of (bilateral) ideals of any ring $R$ is a semiring under ideal sum and product of ideals. This semiring is lattice-ordered by inclusion. The set $M_{n}(\ell(R))$ of $n \times n$ matrices with entries in $d(R)$ is again a semiring under the obvious sum and product operations, also lattice-ordered by entry-wise inclusion: lattice join coincides with semiring sum, denoted + , while the lattice meet operation $\wedge$ is entrywise intersection. The lattice $M_{n}(d(R))$ is complete, it is isomorphic to the $n^{2}$-th Cartesian power of the complete lattice $d(R)$. The matrix $\mathbf{I}$, with the improper ideal $(1)=R$ in diagonal positions and the trivial ideal (0) in off-diagonal positions, is multiplicatively neutral in the semiring $M_{n}(\mathcal{Q}(R))$.

For every matrix $\mathbf{U}$ with ideal entries, $\mathbf{U} \in M_{n}(\mathcal{Q}(R))$, consider the set of matrices

$$
G=\left\{A \in M_{n}(R): \forall i, j \quad A(i, j) \in \mathbf{U}(i, j)\right\}
$$

This set is always an additive subgroup of $M_{n}(R)$, and it is a subring if and only if $\mathbf{U}^{2}+\mathbf{I} \leq \mathbf{U}$ in the ordered semiring of $n \times n$ matrices with ideal entries. In that case, we say that the subring $G$ is defined by $\mathbf{U}$.

There is a natural lattice embedding from the lattice of all pre-orders on $\mathbf{n}$ into the lattice $M_{n}(\mathcal{Q}(R))$, namely the map $\theta \mapsto \mathbf{U}$ where $\mathbf{U}(i, j)$ is the improper or trivial ideal of $R$ according to whether the relation $i \theta j$ holds or not. The matrix $\mathbf{U}$ so obtained always satisfies $\mathbf{U}^{2}+\mathbf{I} \leq \mathbf{U}$, and the subring of $M_{n}(R)$ defined by it coincides with the structural matrix ring $M_{n}(\theta, R)$. For this reason we denote by $M_{n}(R, \mathbf{U})$ the matrix ring defined by any $\mathbf{U} \in M_{n}(\alpha(R))$ satisfying $\mathbf{U}^{2}+\mathbf{I} \leq \mathbf{U}$. Any 
$\mathbf{U} \in M_{n}(\ell(R))$ is viewed as an $\ell(R)$-valued relation on the $n$-element set $\mathbf{n}$, the inequality $\mathbf{U}^{2}+\mathbf{I} \leq \mathbf{U}$ generalizes transitivity and reflexivity of relations, and $M_{n}(R, \mathbf{U})$ may then be thought of as the incidence algebra of the generalized pre-order $\mathbf{U}$.

Matrices with ideal entries $\mathbf{U} \in M_{n}(d(R))$ satisfying the generalized transitivity condition $\mathbf{U}^{2} \leq \mathbf{U}$ only were used by van Wyk [10], and recently again by Meyer, Szigeti and van Wyk [7], in the description of ideals of stuctural matrix rings .

Any matrix with ideal entries $\mathbf{U} \in M_{n}(\Omega(R))$ satisfying $\mathbf{U}^{2}+\mathbf{I} \leq \mathbf{U}$ is said to be reflexive-transitive. If $R$ is a division ring, then the range of the map $\theta \mapsto \mathbf{U}$ described above, embedding the pre-order lattice into $M_{n}(\mathcal{}(R))$, is exactly the set of reflexivetransitive matrices.

For any ring $R$, the set of reflexive-transitive matrices with ideal entries constitutes a complete lattice under the ordering of $M_{n}(I(R))$ (but not a sublattice of $M_{n}(I(R)$ ) in general).

Proposition 4. For any ring $R$, the map $\mathbf{U} \mapsto M_{n}(R, \mathbf{U})$ establishes a lattice isomorphism between:

(i) the lattice $\left\{\mathbf{U} \in M_{n}(\alpha(R)): \mathbf{U}^{2}+\mathbf{I} \leq \mathbf{U}\right\}$ of $n \times n$ reflexive-transitive matrices with ideal entries,

(ii) the lattice of subrings of $M_{n}(R)$ containing all diagonal matrices.

Proof. Obviously if $\mathbf{U}^{2}+\mathbf{I} \leq \mathbf{U}$ then $M_{n}(R, \mathbf{U})$ contains all diagonal matrices, and if $\mathbf{U} \subseteq \mathbf{V}$ then $M_{n}(R, \mathbf{U}) \subseteq M_{n}(R, \mathbf{V})$.

Conversely, let $N \subseteq M_{n}(R)$ be any matrix ring including all diagonal matrices. For every $1 \leq i, j \leq n$ the set $U_{i j}=\{A(i, j): A \in N\}$ is an ideal of $R$, the matrix $\mathbf{U}=\left(U_{i j}\right)$ with ideal entries can be seen to satisfy $\mathbf{U}^{2}+\mathbf{I} \leq \mathbf{U}$, and $M_{n}(R, \mathbf{U})=$ $N$.

The proof above is presented in [5] in somewhat greater detail, together with some consequences. For division rings, where there is only the trivial and the improper ideal, the structure of the lattice of subrings of $M_{n}(R)$ containing all diagonal matrices is independent of the choice of the particular division ring $R$. In the general case, it is the ideal lattice structure of $R$ that determines the structure of this upper section of the subring lattice of $M_{n}(R)$.

\section{REFERENCES}

[1] P. M. Cohn, "Reversible rings," Bull. London Math. Soc., vol. 31, no. 6, pp. 641-648, 1999.

[2] S. Dăscălescu and L. van Wyk, "Do isomorphic structural matrix rings have isomorphic graphs?" Proc. Amer. Math. Soc., vol. 124, no. 5, pp. 1385-1391, 1996.

[3] R. B. Feinberg, "Polynomial identities of incidence algebras," Proc. Amer. Math. Soc., vol. 55, no. 1 , pp. 25-28, 1976.

[4] S. Foldes and G. Meletiou, "On incidence algebras and triangular matrices," Rutcor Res. Report, vol. 2002, no. 35, 2002. [Online]. Available: http://rutcor.rutgers.edu/pub/rrr/reports2002/ 35_2002.pdf 
[5] S. Foldes and G. Meletiou, "On matrix rings containing all diagonal matrices," Tampere University of Technology, 2007. [Online]. Available: http://math.tut.fi/algebra/

[6] S. Foldes, J. Szigeti, and L. v. Wyk, "Invertibility and Dedekind finiteness in structural matrix rings," Linear and Multilinear Algebra, vol. 59, no. 2, pp. 221-227, 2011. [Online]. Available: http://dx.doi.org/10.1080/03081080903357653

[7] J. Meyer, J. Szigeti, and L. van Wyk, “On ideals of triangular matrix rings," Period. Math. Hungar, vol. 59, no. 1, pp. 109-115, 2009.

[8] G.-C. Rota, "On the foundations of combinatorial theory. I. theory of Möbius functions," Z. Wahrscheinlichkeitstheorie und Verw. Gebiete, vol. 2, pp. 340-368, 1964.

[9] J. Szigeti and L. van Wyk, "Subrings which are closed with respect to taking the inverse," $J$. Algebra, vol. 318, no. 2, pp. 1068-1076, 2007.

[10] L. van Wyk, "Special radicals in structural matrix rings," Comm. Algebra, vol. 16, no. 2, pp. 421-435, 1988.

[11] L. van Wyk, "Matrix rings satisfying column sum conditions versus structural matrix rings," Linear Algebra Appl., vol. 249, pp. 15-28, 1996.

Authors' addresses

Stephan Foldes

Tampere University of Technology, PL 553, 33101 Tampere, Finland

E-mail address: sfetut.fi

\section{Gerasimos Meletiou}

TEI of Epirus, PO Box 110, 47100 Arta, Greece

E-mail address: gmelet@teiep.gr 